\title{
CAMBIOS Y CONTINUIDADES EN LA INCORPORACIÓN ESPACIAL DE LOS MIGRANTES PERUANOS EN LA CIUDAD DE CÓRDOBA, ARGENTINA
}

\author{
Changes and Continuities in the Spatial Incorporation of Peruvian \\ Migrants in the city of Cordoba, Argentina
}

\author{
Pablo Sebastián Gómez* \\ David Sanchez Soria**
}

\begin{abstract}
Resumen. La discusión sobre la incorporación de los migrantes en sociedades receptoras es un tema con una vasta trayectoria en los estudios académicos. En este trabajo focalizamos nuestra atención sobre la dimensión espacial en el análisis de la incorporación de los migrantes peruanos en la Ciudad de Córdoba en el periodo 2001-2010. En primer lugar, analizamos los patrones de segregación espacial de la población nativa y de la población peruana. En segundo lugar, considerando el clivaje concentración/dispersión, analizamos las características sociodemográficas de la población peruana agrupada en clústers y la que se encuentra dispersa. Se sugiere la interacción de dos procesos: a) la existencia de patrones específicos de localización de la migración peruana en la Ciudad de Córdoba. La población nativa con niveles educativos alto, por su parte, tiende a concentrarse en la zona centro y noroeste de la ciudad. En esos espacios centrales se localizan clústers de la comunidad peruana, sin embargo, la evolución histórica señala nuevos asentamientos en zonas periféricas; b) diferencias estructurales entre los peruanos agrupados en clústers y aquellos dispersos.
\end{abstract}

Palabras clave: incorporación, migrantes, estadística espacial, segregación, peruanos.

\begin{abstract}
Debates on the incorporation of migrants in receiving societies is a controversial issue in academic research. In this paper we focus our attention on the spatial dimension in the analysis of the patterns of incorporation of Peruvian migrants in the city of Cordoba in the period of 2001-2010. First, we analyze patterns of spatial segregation of the native population and the Peruvian population in the city. Second, considering the clustered/scattered

* Consejo Nacional de Investigaciones Científicas y Técnicas (CONICET), Universidad Nacional de Córdoba (UNC) y Universidad Siglo 21. Córdoba, Argentina.

** Asociación Ecuatoriana de Estudios de Población (AEEP)/Centro de Estudios Avanzados/
\end{abstract} Universidad Nacional de Córdoba. Córdoba, Argentina. 
cleavage, we analyze the sociodemographic characteristics of clustered and dispersed peruvians in the city. We suggest the interaction of two processes of spatial incorporation: a) there are specific patterns of location of Peruvian migrants in the city of Cordoba. The native population with higher education levels, in turn, tends to settle in the center and northwest part of the city. The central areas were the stable places where the Peruvians are located, however, in recent years there has been a changing pattern with new places of settlement; b) we identify structural differences between clustered and dispersed Peruvians.

Keywords: incorporation, migrants, spatial statistics, Peruvians.

\section{Introducción}

La discusión sobre la incorporación de los migrantes en sociedades receptoras es un tema con una vasta trayectoria en los estudios académicos en diferentes partes del mundo. Las investigaciones analizaron diferentes dimensiones con una gran pluralidad teórica-metodológica. De manera específica, el análisis sobre los patrones residenciales de los grupos migratorios y étnicos tiene una larga historia, especialmente cuando consideramos la migración hacia los Estados Unidos, donde sociólogos y geógrafos urbanos mapearon a los diferentes grupos y analizaron los niveles de segregación y concentración ${ }^{1}$. Los estudios de este tipo recorren un largo camino metodológico para medir la segregación con diferentes índices en el análisis de los niveles y patrones (índices de aislamiento, índice de entropía, índice de interacción, índice de disimilitud). El clásico trabajo de Massey y Denton ${ }^{2}$ sobre las cinco dimensiones de la segregación (igualdad, exposición, concentración, centralidad y agrupamiento) va precisamente en dirección a la generación de indicadores que midan el grado y la intensidad de estos procesos. Sin embargo, este tipo de indicadores no consideran el espacio en términos de representación ${ }^{3}$. En el contexto de Argentina los trabajos que consideran al espacio como una dimensión analítica y con un abordaje cuantitativo son múltiples en los últimos años. Para el periodo de las migraciones de Europa el trabajo de Otero y Pellegrino ${ }^{4}$ analiza las pautas residenciales de los migrantes en las ciudades de Buenos Aires y Montevideo. En ese periodo gran parte de la evidencia empírica analizada que recibió mayor atención fue el análisis de los matrimonios y las asociaciones voluntarias, concitando menor atención el análisis

1 BOLT, Gideon, ÖZÜEKREN, Sule, PHILLIPS, Deborah. Linking Integration and Residential Segregation.

2 MASSEY, Douglas, DENTON, Nancy. The Dimensions of Residential Segregation.

3 La diferencia es que las teorías estadísticas tradicionales basan sus modelos en observaciones independientes, por el contrario, la estadística espacial se basa en la hipótesis de la no independencia de las observaciones.

4 OTERO, Hernan, PELLEGRINO, Adela. Compartir la ciudad. Patrones de residencia e integración de inmigrantes en Buenos Aires y Montevideo durante la inmigración masivas. 
de las pautas residenciales debido a las "dificultades metodológicas" de trabajar con ese tipo de indicadores. En el caso específico de Córdoba y en el periodo denominado de las "migraciones masivas", el pionero trabajo de Szchuman ${ }^{6}$ analiza la movilidad social y las pautas residenciales de los migrantes (sin embargo no utiliza herramientas de estadística espacial como tampoco índices de segregación). Szuchman ${ }^{7}$ analiza la inserción laboral, residencial, educacional y económica de los migrantes europeos en el periodo 1869-1909. El autor señala las diferencias existentes en la incorporación de los migrantes europeos en relación a Buenos Aires y cuestiona la interpretación germaniana sobre una nación fusionada, señalando los "límites del crisol de razas" en la ciudad de Córdoba. Si el "crisol de razas" existió, no fue universal y no todos los grupos étnicos tuvieron los mismos patrones de "fusión". En la actualidad el panorama migratorio cambia y el foco de atención está en el estudio de los procesos de migraciones regionales desde países vecinos hacia la Argentina. Los trabajos que exploraron la dimensión espacial de los migrantes son múltiples en los últimos años. Para la Ciudad Autónoma de Buenos Aires, las investigaciones señalan que el flujo de Perú tiene características diferenciales: se encuentra más disperso en la Ciudad, se localiza más cerca del centro urbano y comparativamente (en relación a los otros grupos migratorios de países limítrofes) se destaca un patrón de asentamiento espacial no vinculado con áreas empobrecidas de la ciudad lo que implica otra relación con el acceso al suelo ${ }^{8}$. En relación a los migrantes de países limítrofes en la Ciudad de Córdoba es de destacar como antecedente el trabajo de Molinatti y Peláez ${ }^{9}$ que utiliza herramientas de estadística espacial para analizar la segregación residencial de migrantes peruanos y bolivianos en la ciudad de Córdoba para los años 2001 y 2008.

En este trabajo queremos focalizar nuestra atención en los procesos migratorios que ocurren entre los países del sur global en general y la migración peruana en particular en la Ciudad de Córdoba, Argentina. La migración peruana en la ciudad es el flujo latinoamericano más importante en términos cuantitativos seguido en relevancia por la migración boliviana. En comparación a las migraciones sur-norte, estos flujos presentan especificidades tanto en la

5 DEVOTO, Fernando, OTERO, Hernan. Veinte años después: una lectura sobre el Crisol de razas, el pluralismo cultural y la historia nacional en la historiografía argentina.

6 SZUCHMAN, Marck. Mobility and Integration in Urban Argentina: Córdoba in the Liberal Era.

7 Ibidem.

8 MERA, Gabriela, MARCOS, Mariana. Cartografías migratorias urbanas. Distribución espacial de la población extranjera en la Ciudad de Buenos Aires; MERA, Gabriela, MARCOS, Mariana, VIRGILIO, María Mercedes. Migración internacional en la Ciudad de Buenos Aires: un análisis socioespacial de su distribución según tipos de hábitat; MARCOS, Mariana, MERA, Gabriela. Migrantes internacionales en la Aglomeración Gran Buenos Aires: un análisis cuantitativo de su distribución espacial.

9 MOLINATTI, Florencia, PELAEZ, Enrique Peláez. Segregación residencial y migrantes de Bolivia y del Perú. Niveles y patrones espaciales en la ciudad de Córdoba a comienzos del siglo XX. 
composición de clase de los mismos como en el contexto normativo y social del espacio de recepción.

En ese contexto en este trabajo analizamos los patrones de incorporación espacial de la población nacida en Perú en la Ciudad de Córdoba, Argentina en el periodo 2001-2010. De manera específica, en primer lugar, analizamos los patrones de segregación espacial de la población nativa y de la población peruana en la ciudad. En segundo lugar, considerando la dimensión espacial como clivaje, analizamos las características sociodemográficas de la población peruana agrupada en clústers y la que se encuentra dispersa en la Ciudad.

\section{La incorporación espacial de los migrantes y la migración}

\section{peruana}

Fundamentalmente en el contexto de Estados Unidos uno de los modelos teóricos para explicar los patrones de asentamiento residencial de los grupos étnicos/migratorios fue el de la asimilación espacial ${ }^{10}$.

El clásico modelo de la asimilación espacial ${ }^{11}$ postulaba que los grupos migratorios tienden a movilizarse hacia barrios "blancos" como parte de un proceso más amplio de "asimilación" ${ }^{12}$ cultural y socioeconómica. Los migrantes generalmente llegan a través de cadenas migratorias y en función de eso tienden a vivir en enclaves étnicos donde obtienen ayuda de sus connacionales. A medida que el proceso de aculturación ocurre, las preferencias habitacionales cambiarían buscando espacios de la ciudad con mayor status socioeconómico. Es decir, según esta teoría, el proceso de aculturación provee el deseo y la movilidad social los medios para que los migrantes se asimilen espacialmente. De manera general los miembros de los grupos migratorios gradualmente se incorporan a la cultura dominante y van perdiendo progresivamente sus características étnico/nacionales. Cuando este modelo de la asimilación considera el espacio, se sugiere que la movilidad social también implicaría una fusión en términos espaciales con los patrones de la sociedad de destino. De manera específica, la segregación residencial de los grupos migratorios con el paso del tiempo tendería a disminuir en tanto que el status socioeconómico de los migrantes aumenta. El proceso secuencial implica que las minorías

\footnotetext{
${ }^{10}$ SOUTH, Scott, CROWDER, Kyle, CHAVEZ, Erick. Migration and spatial assimilation among U.S. latinos: Classical versus segmented trajectories; MASSEY, DENTON, op. cit.; BOLT, ÖZÜEKREN, PHILLIPS, op. cit.; FREEMAN, Lance. Minority Housing Segregation: A Test of Three Perspectives; FREEMAN, Lance. Does Spatial Assimilation Work for Black Immigrants in the US?; GALSTER, George. The Evolving Challenges of Fair Housing Since 1968: Open Housing, Integration, and the Reduction of Ghettoization.

${ }^{11}$ PARK, Robert. The Urban Community as a Spatial Pattern and a Moral Order; GORDON, Milton. Assimilation in American Life: The Role of Race, Religion and National Origins.

12 Para una genealogía del concepto de asimilación véase KIVISTO, Peter. What is the canonical theory of assimilation?.
} 
étnicas se concentren en específicas áreas de la ciudad debido a las redes sociales establecidas y los potenciales beneficios del capital social. A medida que existe un proceso de asimilación cultural y socioeconómica también se produce una asimilación en términos del espacio urbano ${ }^{13}$. Los migrantes más recientes deberían tener niveles mayores de segregación que los que ya están establecidos y las segundas generaciones estarán más fusionadas. Sería algo deseable, según este modelo analítico, dejar los espacios de concentración migratoria, posibilitado por la movilidad socioeconómica. El componente clave en esta dinámica está dado por un elemento central: las sucesivas generaciones son el motor de cambio hacia una mayor fusión con los patrones de la sociedad de destino. La clásica teoría de la asimilación en sus aspectos generales fue objeto de profundas críticas ${ }^{14} .{ }^{15}$

Estos modelos explicativos tuvieron como centro de gravitación lo ocurrido en el contexto de Estados Unidos. Sin embargo, es problemático cuando analizamos otros contextos de manera general y de manera específica las migraciones sur-sur en América Latina, por lo que debemos ser cautos cuando aplicamos acríticamente estos esquemas analíticos. Por ejemplo, para el caso europeo se señala que los niveles de segregación son relativamente bajos y los niveles de integración relativamente altos mientras que en Estados Unidos los niveles de segregación son relativamente altos y los niveles de integración bajos ${ }^{16}$. Consideramos que la temática de la segregación/dispersión en el espacio urbano de los migrantes no puede ser analizada como un fenómeno aislado de la sociedad en su conjunto y se comprende como el resultado de múltiples procesos (por ejemplo: los patrones de segregación que existen en la ciudad de la población en su conjunto). En este trabajo consideramos que hay concentración espacial si un área de la ciudad presenta una sobrerrepresentación de ciertos grupos migratorios (comparado con la distribución del grupo en la ciudad en su conjunto) ${ }^{17}$.

La migración peruana en Argentina experimentó un crecimiento sostenido en los últimos años. La evolución de los valores absolutos pasa de 8.002 en 1980 a 16.548 en 1991 y de 88.260 en el 2001 a 157.514 en el último censo del 2010. El Gran Buenos Aires es el lugar de mayor concentración de este grupo migratorio

\footnotetext{
13 ALBA, Richard, NEE, Victor. Remaking the American mainstream: assimilation and contemporary immigration; BOLT, Gideon, KEMPEN, Ronald. Escaping poverty neighbourhoods in the Netherlands.

${ }^{14}$ Vease JUNG, Moon-Kie. The Racial Unconscious of Assimilation Theory.

${ }^{15}$ Este modelo explicativo fue objeto de críticas. Los limites a la incorporación espacial fueron señalados por la teoría de la estratificación de lugares y el modelo del enclave étnico. Para más detalle véase FREEMAN, Minority..., op. cit.; FREEMAN, Does Spatial..., op. cit.

${ }^{16}$ MUSTERD, Sako. Segregation and integration: A contested relationship.

17 KEMPEN, Ronald, ÖZÜEKREN, Sule. Ethnic Segregation in Cities: New Forms and Explanations in a Dynamic World.
} 
y una de sus características principales que comparte con el resto de los migrantes limítrofes es que mantienen una inserción relativamente marginal en el mercado de trabajo y se concentran sobre todo en los sectores de mayor informalidad y precariedad laboral (como la construcción, la industria textil de confección y calzado, comercio al por menor y comercio doméstico) ${ }^{18}$.

Si bien Córdoba no es el principal destino de la migración peruana, presenta especificidades que ameritan su detallado estudio. La población peruana residente en Córdoba pasa de 6.750 personas en el 2001 a 12.442 en el 2010 concentrando casi el $8 \%$ del total de los peruanos residentes en la Argentina. El crecimiento intercensal de los peruanos en Córdoba es levemente superior al experimentado para el total de la Argentina ${ }^{19}$.

A partir de estos esquemas analíticos y las investigaciones previas tomamos como hipótesis inicial de trabajo que existe una incorporación espacial, entendida como un proceso donde inicialmente los grupos migratorios tienden a concentrarse en determinadas zonas de la ciudad y que esa característica posibilita beneficiarse del grupo de connacionales. Las investigaciones realizadas en la Ciudad de Córdoba sobre la migración peruana destacan la importancia de las redes sociales y el papel de las mujeres en el proceso migratorio ${ }^{20}$.

Con el transcurso del tiempo hay un proceso de progresiva dispersión espacial e incorporación a la estructura de clases de la sociedad de destino. Las investigaciones para otros contextos migratorios de migración peruana indican un proceso similar: en Estados Unidos se señala que los migrantes tienden a dispersarse espacialmente hacia nuevos destinos y que eso se refleja en el acelerado crecimiento relativo de la población observada en zonas que tradicionalmente no albergaban comunidades migrantes ${ }^{21}$.

Sin embargo, aquí queremos agregar una serie de hipótesis adicionales: a) la dispersión en el espacio urbano no implica necesariamente una convergencia con los patrones ocupacionales de la población nativa (se necesita más investigación empírica sobre este tópico para el caso de la Ciudad de Córdoba); b) la dispersión espacial implica la convergencia hacia los mismos patrones de segregación espacial de la población nativa (y por lo tanto están sujetos a las mismas dinámicas de desigualdad en el acceso al suelo, vivienda, servicios públicos, etc.); c) en función de lo anterior, enfatizamos la relevancia de la

${ }^{18}$ CERRUTTI, Marcela, MAGUID, Alicia. Inserción laboral e ingresos de los migrantes de países limítrofes y peruanos en el gran Buenos Aires.

19 INDEC. Censo Nacional de Población, Hogares y Viviendas del año 2001; INDEC. Censo Nacional de Población, Hogares y Viviendas del año 2010.

${ }^{20}$ BAIADERA ALVITES, Angelica. Entre redes sociales. Prácticas y representaciones de la migración peruana. Córdoba, Argentina; MAGLIANO, Maria, PERISSINOTTI, Maria, ZENKLUSEN, Denise. Mujeres bolivianas y peruanas en la migración hacia Argentina: especificidades de las trayectorias laborales en el servicio doméstico remunerado en Córdoba.

${ }^{21}$ MORENO, Martín. La distribución espacial de las comunidades peruanas en los Estados Unidos. 
composición del flujo migratorio, donde la clase social es un factor relevante para comprender las condiciones de dispersión espacial. En las investigaciones previas $^{22}$ en la ciudad de Córdoba se señala la existencia de dos macro-cohortes de migrantes peruanos: 1) aquellos migrantes que arribaron a partir de la década del sesenta y cuya motivación fue esencialmente la capacitación profesional; 2) migrantes recientes que llegaron principalmente en la década de 1990 y cuya motivación fue esencialmente laboral.

Las investigaciones previas ${ }^{23}$ que analizaron los patrones para el censo 2001 consideraron el clivaje concentración/dispersión en el espacio urbano de los migrantes peruanos y se encontró que los migrantes peruanos hombres que no están agrupados tienen mayor diversificación en cuanto a la estructura ocupacional y una mejor posición socioeconómica que los hombres agrupados. Las mujeres por el contrario presentaron una situación socioeconómica más precaria que los hombres, tanto las que se encontraban dispersas como agrupadas. Estos trabajos señalaron la relevancia de comprender la composición de clase del flujo migratorio, el género y la temporalidad a través de las macro cohortes del flujo migratorio. Sin embargo, no se analizaron los cambios y continuidades en los últimos veinte años de los patrones espaciales de los migrantes peruanos (fechas de los últimos censos) ni la convergencia o no con los patrones espaciales de la población nativa.

\section{Datos y Metodología}

Para analizar los cambios y las continuidades en la incorporación espacial de los migrantes peruanos en la Ciudad de Córdoba utilizamos datos censales de la República Argentina, específicamente los censos correspondientes a los años 2001 y 2010 del Instituto Nacional de Estadísticas y Censos (INDEC), Argentina.

El Censo Nacional de Población, Hogares y Viviendas 2001 fue un censo de hecho, modalidad histórica de relevamiento censal en Argentina. Es decir, se censaron a las personas en el hogar que pasaron la noche de referencia del censo (del 16 al 17 de noviembre) fueran o no residentes habituales. Es importante destacar que el censo 2001, a diferencia de los Censos de 1980 y 1991, no aplicó una muestra en el relevamiento sino que se formularon las mismas preguntas a toda la población, sin excepción, mediante la aplicación de un cuestionario único.

\footnotetext{
${ }^{22}$ FALCÓN, Maria, BOLOGNA, Eduardo. Migrantes antiguos y recientes: Una perspectiva comparada de la migración peruana a Córdoba, Argentina; BOLOGNA, Eduardo, FALCÓN, Maria. Migración sur-sur: Factores relacionales e inserción segmentada de la población boliviana y peruana en la ciudad de Córdoba (Argentina).

${ }^{23}$ GÓMEZ, Pablo Sebastian, SANCHEZ SORIA, David. Concentración, dispersión y características sociodemográficas en la incorporación espacial de la migración peruana en la Ciudad de Córdoba, Argentina.
} 
El Censo Nacional de Población, Hogares y Viviendas del año 2010 también fue un censo de hecho. Es decir que fueron censadas las personas que se encontraron presentes en la vivienda censada a la hora cero del 27 de octubre, fueran residentes habituales o no. Sin embargo, a diferencia del censo del 2001 en este operativo censal, al igual que los de los años 1980 y 1991 se aplicó una metodología con muestra. Es decir, en las viviendas donde las personas viven bajo un régimen familiar (viviendas particulares) se aplicó un cuestionario ampliado o uno básico. El cuestionario ampliado se aplicó a todas las localidades de menos de 50.000 habitantes y a las localidades mayores de esa cantidad se tomó una muestra del $10 \%$ de las viviendas, al resto de las viviendas de estas localidades se aplicó el cuestionario básico. Este dato es relevante para nuestros objetivos de investigación ya que implicó que algunas variables analizadas en el censo 2001 no estuvieran disponibles en el censo 2010 para su georreferenciación.

En la primera etapa nos proponemos analizar la incorporación en el espacio urbano de los migrantes peruanos y si existe algún patrón común en relación a la población nativa. Analizamos la distribución en el espacio urbano de dos variables de análisis: la nacionalidad peruana según el lugar de nacimiento y el nivel educativo alto del conjunto de la población nativa (consideramos el nivel educativo un proxy del nivel socioeconómico). El nivel alto de esta variable corresponde a los individuos con más de doce años de escolarización en el censo 2001 y a los niveles: superior no universitario, universitario y postuniversitario en el censo 2010. Debemos señalar que ninguno de los censos tiene información sobre ingresos y por eso decidimos considerar la variable nivel educativo.

Aplicamos dos tipos de técnicas para analizar la distribución espacial de la población nacida en Perú y la población nativa con nivel educativo alto: 1) medidas globales de autocorrelación espacial (Índice Global de Moran) y 2) medidas locales de agrupación espacial para representar clústers de población (Gi* de Getis Ord).

El índice global de Moran propuesto por Getis y $\operatorname{Ord}^{24}$ mide la autocorrelación espacial basada en las ubicaciones y los valores de las entidades simultáneamente. Este índice evalúa si el patrón de un atributo específico en un conjunto de entidades, está agrupado, disperso o es aleatorio. Sus valores varían entre -1 y 1 , donde los valores positivos confirman la existencia de correlación espacial.

Los cálculos se realizaron en el software ArcGis 10.2. Al tratarse de un indicador global, este representa una estadística resumen de la intensidad de la auto-correlación entre grupos de unidades territoriales, pero no identifica

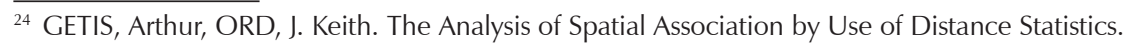


clústers o patrones espaciales. Esa dimensión es analizada con el índice Gi* de Getis Ord. Dado que esta técnica se refiere a un test espacial global-es decir analiza todas las observaciones de forma conjunta por lo que no es sensible a casos donde predomine una inestabilidad de la distribución espacial de los datos- requiere del cálculo de estadísticos locales de asociación espacial, como $\mathrm{Gi}^{*}$ de Ord y Getis ${ }^{25}$.

La estadística Gi* de Getis y Ord realiza la búsqueda de cada unidad de análisis en el contexto de entidades vecinas. Una entidad con un valor alto es interesante, pero es posible que no sea un punto caliente estadísticamente significativo. Para ser un punto caliente estadísticamente significativo, debe tener un valor alto y también estar rodeada por otras entidades con valores altos. La suma local para ésta y sus vecinos se compara proporcionalmente con la suma de todas las entidades; cuando la suma local es muy diferente de la esperada, y esa diferencia es demasiado grande como para ser el resultado de una opción aleatoria, se obtiene como consecuencia una puntuación z estadísticamente significativa.

En la segunda etapa después de detectar clústers de población nacida en Perú y población nativa con nivel educativo alto nos proponemos realizar un análisis sociodemográfico de la población nacida en Perú que se encuentra concentrada en la Ciudad y aquella que se encuentra dispersa para el periodo 2001 y 2010. Para el análisis de las zonas Hot Spot (zonas de concentración) se seleccionó los radios con el valor Gi* Z Score superior a 2, que corresponde las zonas con más del 95\% confianza. Con el objetivo de determinar los radios correspondientes se utilizó una unión espacial y se clasificó a cada clúster.

\section{La incorporación de los migrantes peruanos en la Ciudad de Córdoba}

\subsection{Autocorrelación global}

El primer paso en el análisis fue utilizar el índice global de Moran aplicado a la población de origen peruano y la población nativa con nivel educativo alto. En la tabla 1 se presentan los resultados del cálculo, para los radios censales analizados existen áreas de agrupación no aleatorias. Es decir, rechazamos la hipótesis nula de aleatoriedad en el patrón de distribución de población de personas con nivel educativo alto y también de la población peruana. Hay concentración de población con nivel educativo alto y también concentración de población de origen peruano para ambos periodos analizados.

\footnotetext{
${ }^{25}$ ORD, J. Keith, GETIS, Arthur. Local Spatial Autocorrelation Statistics: Distributional Issues and an Application.
} 


\begin{tabular}{lcccc}
$\begin{array}{l}\text { Tabla } \mathbf{1} \text { - Autocorrelación espacial global de población nacida en Perú } \mathbf{2 0 0 1} \text { y } \\
\mathbf{2 0 1 0} \text { y de población nativa con nivel educativo alto } \mathbf{2 0 0 1} \text { y } \mathbf{2 0 1 0 .}\end{array}$ \\
\hline & $\begin{array}{c}\text { Población } \\
\text { peruana }\end{array}$ & $\begin{array}{c}\text { Población nativa } \\
\text { Niv educ. alto }\end{array}$ & $\begin{array}{c}\text { Población } \\
\text { peruana }\end{array}$ & $\begin{array}{c}\text { Población nativa } \\
\text { Niv educ. alto }\end{array}$ \\
\hline & 0,488854 & 0,72248 & 0,297513 & 0,899967 \\
Índice de moran & $-0,000728$ & $-0,000728$ & $-0,00067$ & $-0,00067$ \\
Índice esperado & 0,000047 & 0,000049 & 0,000037 & 0,000044 \\
Varianza & 71,27 & 103,484 & 48,99 & 135,91 \\
Puntuación z & 0.0000 & 0.00000 & 0.0000 & 0.0000 \\
\hline Valor p & & & &
\end{tabular}

Fuente: elaboración propia con base en datos de INDEC $(2001,2010)$.

\subsection{Auto-correlación local}

A continuación se presentan los resultados de la auto-correlación espacial local de datos para dos grupos de población: a) la segregación espacial de la población total con nivel educativo alto y b) la segregación espacial de la población peruana.

Como puede observarse los mapas 1 y 3 , existe concentración de hogares con jefes de hogar cuyo nivel educativo es alto y ese patrón es estadísticamente significativo. El centro de la ciudad, espacio social caracterizado por la presencia de centros comerciales y otros lugares de circulación propios de la clase media acusa, como es de esperar, un patrón de concentración de hogares con niveles socioeconómicos altos. El noroeste, también concentra los mayores niveles educativos con un anillo periférico en la ciudad que concentra los niveles educativos bajos. Este proceso, como es de suponer, se mantiene estable en los dos periodos censales.

Las investigaciones llevadas a cabo en la Ciudad de Córdoba ${ }^{26}$, señalan que en términos de segregación residencial hay una alta fragmentación con una tendencia en dirección a profundizarse en las últimas décadas. Si bien en el estudio citado se señalan las dificultades de establecer patrones comparativos con relación a otras ciudades latinoamericanas (debido a las escalas de análisis, índices y atributos socioeconómicos), se señala que Córdoba muestra niveles de segregación mayores a otras ciudades de la región.

En los mapas 2 y 4 se presenta el análisis de hotspots o puntos calientes calculados para la población cuyo país de nacimiento es Perú. Como puede observarse en el mapa 2, hay tres espacios de concentración de la población de origen peruano. Merece especial atención el clúster ubicado en el centro de la ciudad, en un espacio que históricamente se caracterizó por la presencia de

${ }^{26}$ MOLINATTI, op. cit. 
peruanos ya desde la década del sesenta, la ubicación de un hospital escuela de la Universidad Nacional de Córdoba y la localización de estudiantes que llegaban de diversos puntos del país. Los otros puntos se encuentran en la zona noroeste de la ciudad y se superponen con los de la población nativa con nivel educativo alto. Como puede observarse en el mapa 4 , se detectaron 4 clústers. Dos en una zona central y dos en una zona periférica de la ciudad. De acuerdo al mapa 4 podemos observar que hay algunos clústers que se encuentran en zonas de concentración de población con nivel educativo alto en tanto que otros en zonas frías (coldspots). Si bien hay cambios en las zonas de concentración de peruanos, hay una estabilidad en la zona céntrica como espacio que condensa la población de origen peruano. El incipiente proceso de gentrificación de esa zona y la persistencia de migrantes peruanos va en dirección de sostener la hipótesis de que las redes sociales siguen materializando los elementos que direccionan el proceso migratorio hacia esa zona de la ciudad. Las investigaciones previas sugieren que casi el $83 \%$ de los migrantes peruanos tenían compatriotas conocidos al momento de su arribo ${ }^{27}$. El clúster periférico en la zona sur de la ciudad, por su parte, se caracteriza por un barrio con alta concentración de migrantes de diversas nacionalidades (peruanos, bolivianos y paraguayos) y está ubicado en particulares condiciones socioambientales. Monayar y Marengo ${ }^{28}$, señalan en su estudio que se trata de un barrio periférico que se consolida a partir de la ocupación informal del suelo y con un alto riesgo ambiental debido a la presencia de un basural antes de la ocupación ${ }^{29}$. Se necesita más investigación empírica para comparar los diferentes clúster de población peruana, objeto de investigación que excede este artículo. Debemos recordar que la zona céntrica de la ciudad se caracteriza por tener mayor acceso a servicios, hospitales y colegios. Sumada a esta característica estructural de la ciudad, existe un sistema de exclusión de hecho en la ciudad que dificulta el acceso a las zonas céntricas de la ciudad de los habitantes de la periferia (por los mecanismos de discriminación y principalmente por la presencia policial que establece "aduanas" difíciles de franquear $)^{30}$.

\footnotetext{
27 FALCÓN, BOLOGNA, op. cit.

${ }^{28}$ MARENGO, Cecilia, MONAYAR, Virginia. Crecimiento urbano e informalidad residencial: El caso Nuestro Hogar III en la periferia de Córdoba, Argentina.

${ }^{29}$ Las autoras Monayar y Marengo (ibidem) también apuntan que fue el resultado de un loteo fraudulento originado en el año 1997 y que consistió en la venta de 1683 lotes por un agente informal del suelo, es decir, sin títulos legales de los mismos.

${ }^{30}$ BOLOGNA, Eduardo et alii. El derecho a la ciudad en cuestión: segregación residencial y experiencias de circulación de jóvenes en la Ciudad de Córdoba, Argentina.
} 


\section{Mapa 1 - Distribución espacial del estadístico Gi* para la población con nivel educativo alto. 2001.}

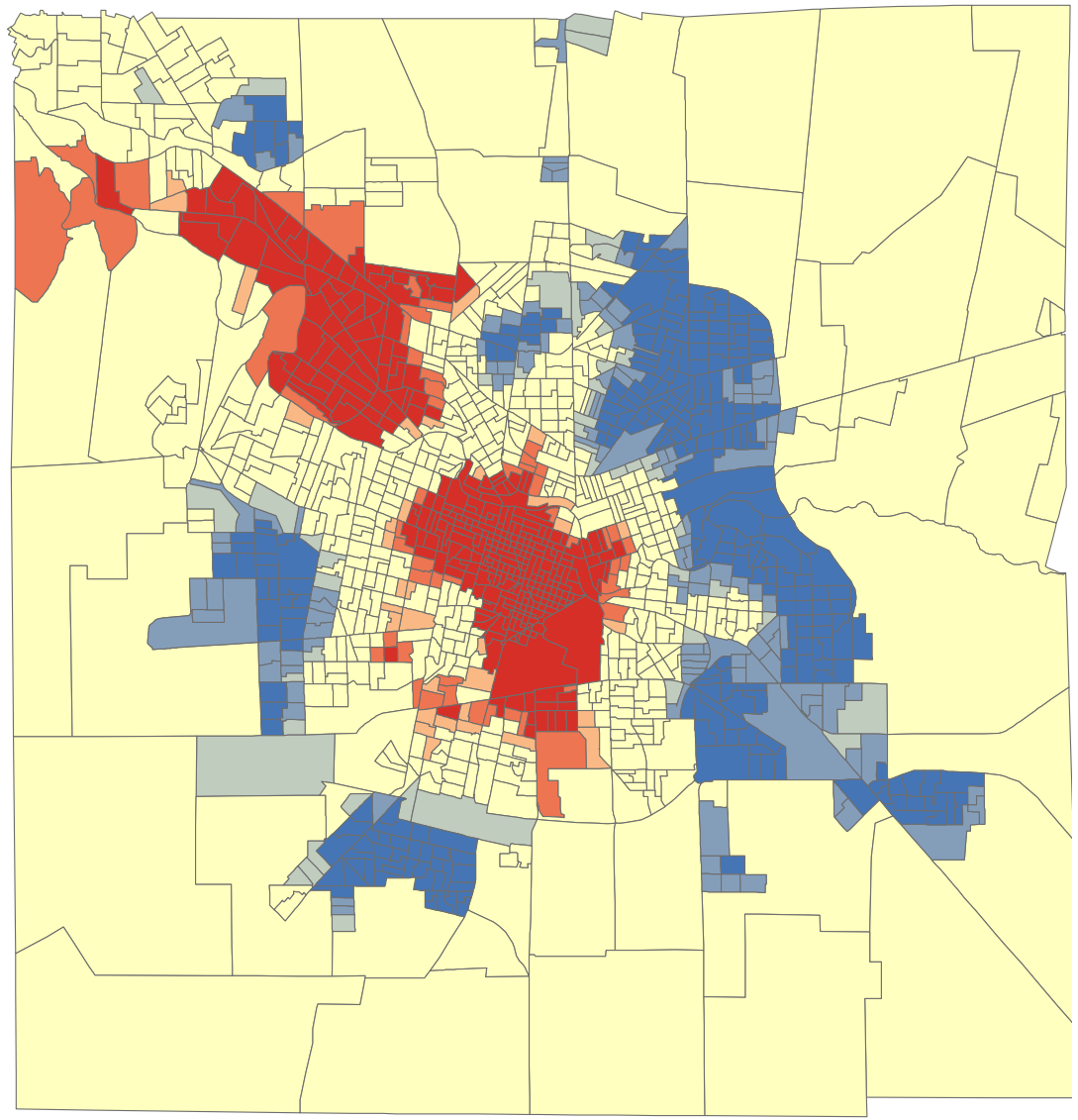

\section{Gi_Bin}

Cold Spot - 99\% Confidence Cold Spot - 95\% Confidence Cold Spot - 90\% Confidence Not Significant Hot Spot - 90\% Confidence Hot Spot - 95\% Confidence Hot Spot - 99\% Confidence

Fuente: elaboración propia con base en datos de INDEC (2001). 


\section{Mapa 2 - Distribución espacial del estadístico Gi* para la población nacida en}

Perú. 2001.
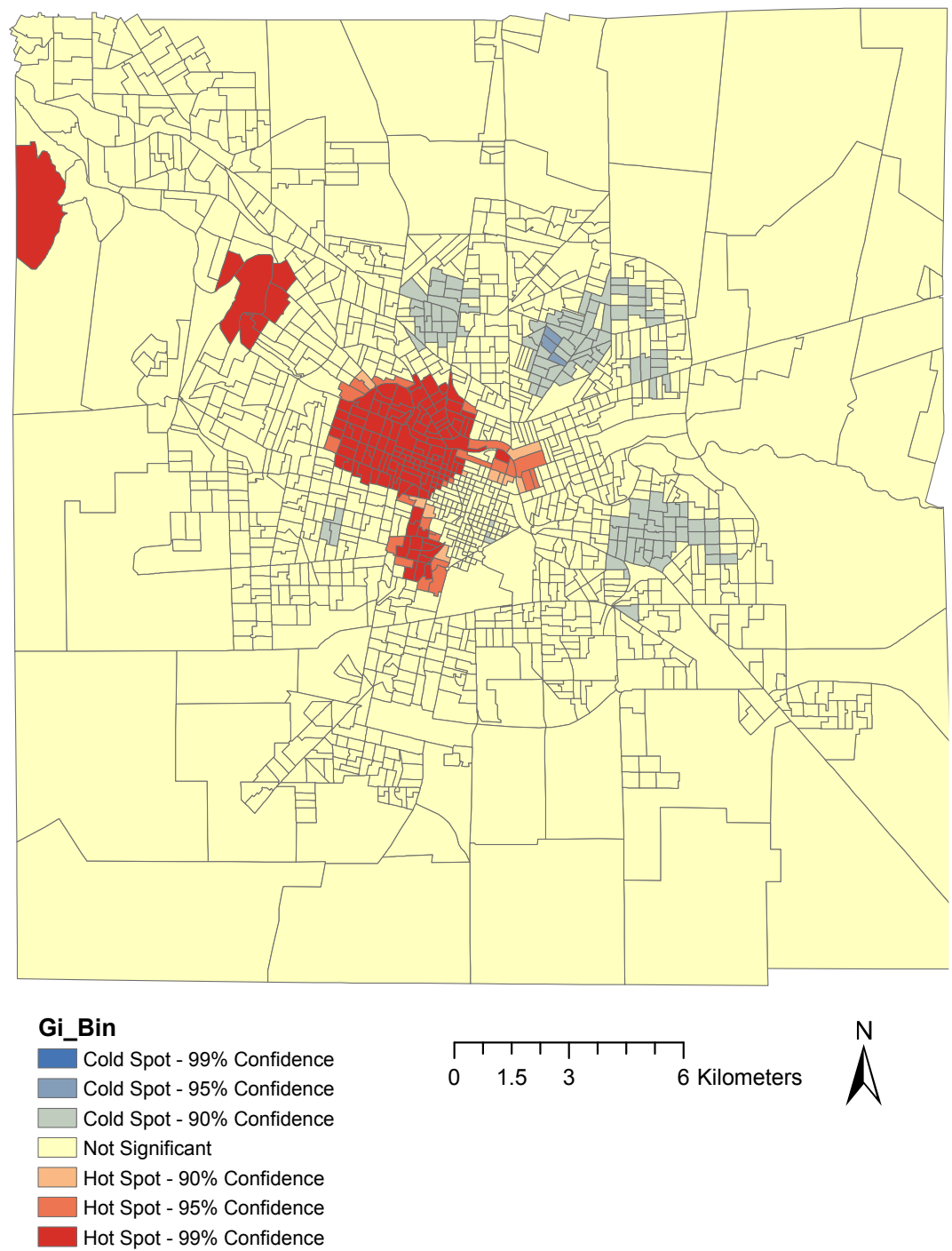

Fuente: elaboración propia con base en datos de INDEC (2001). 


\section{Mapa 3 - Distribución espacial del estadístico Gi* para la población con nivel educativo alto. 2010.}
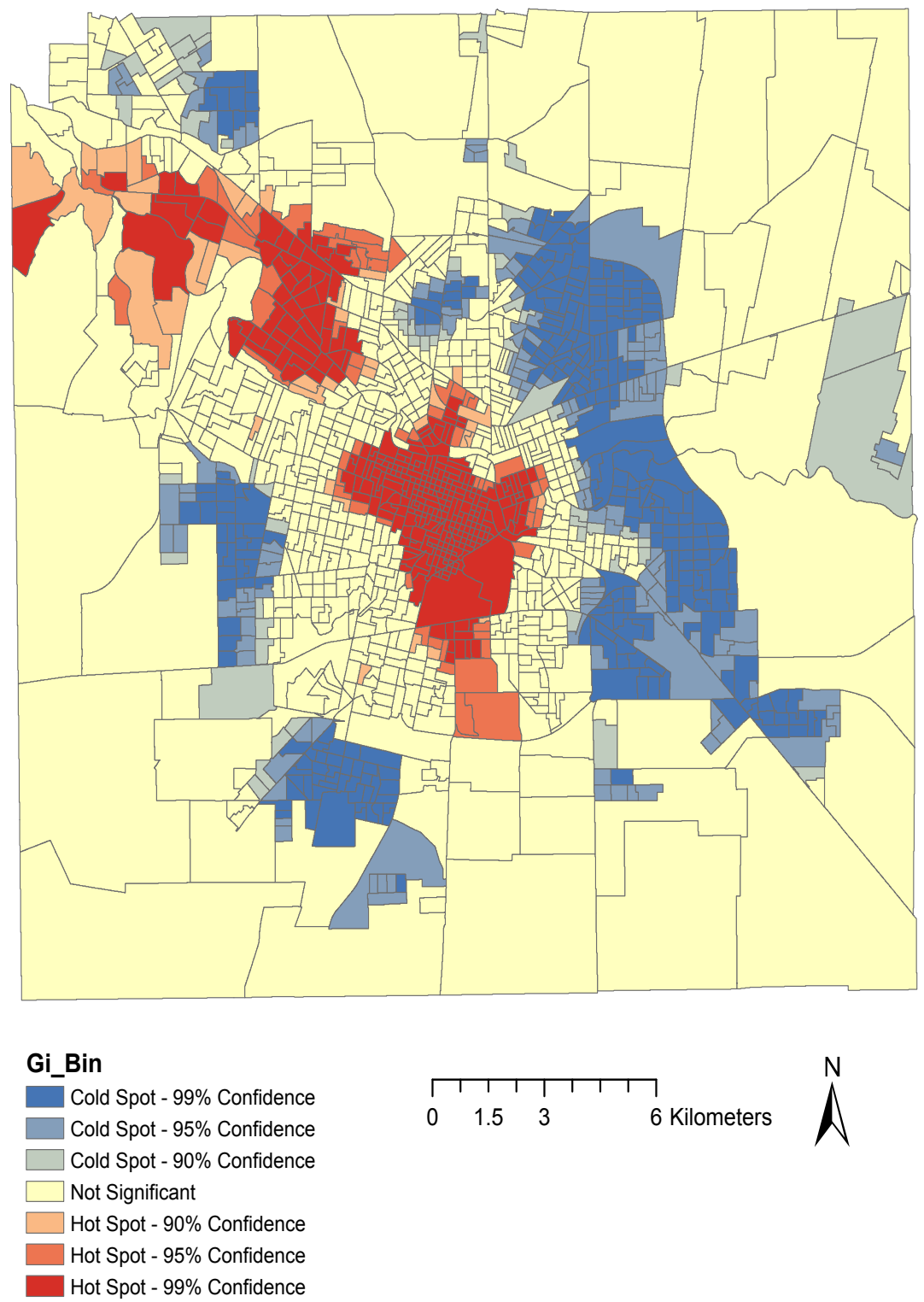

Fuente: elaboración propia con base en datos de INDEC (2010). 


\section{Mapa 4 - Distribución espacial del estadístico Gi* para la población nacida en}

Perú. 2010.

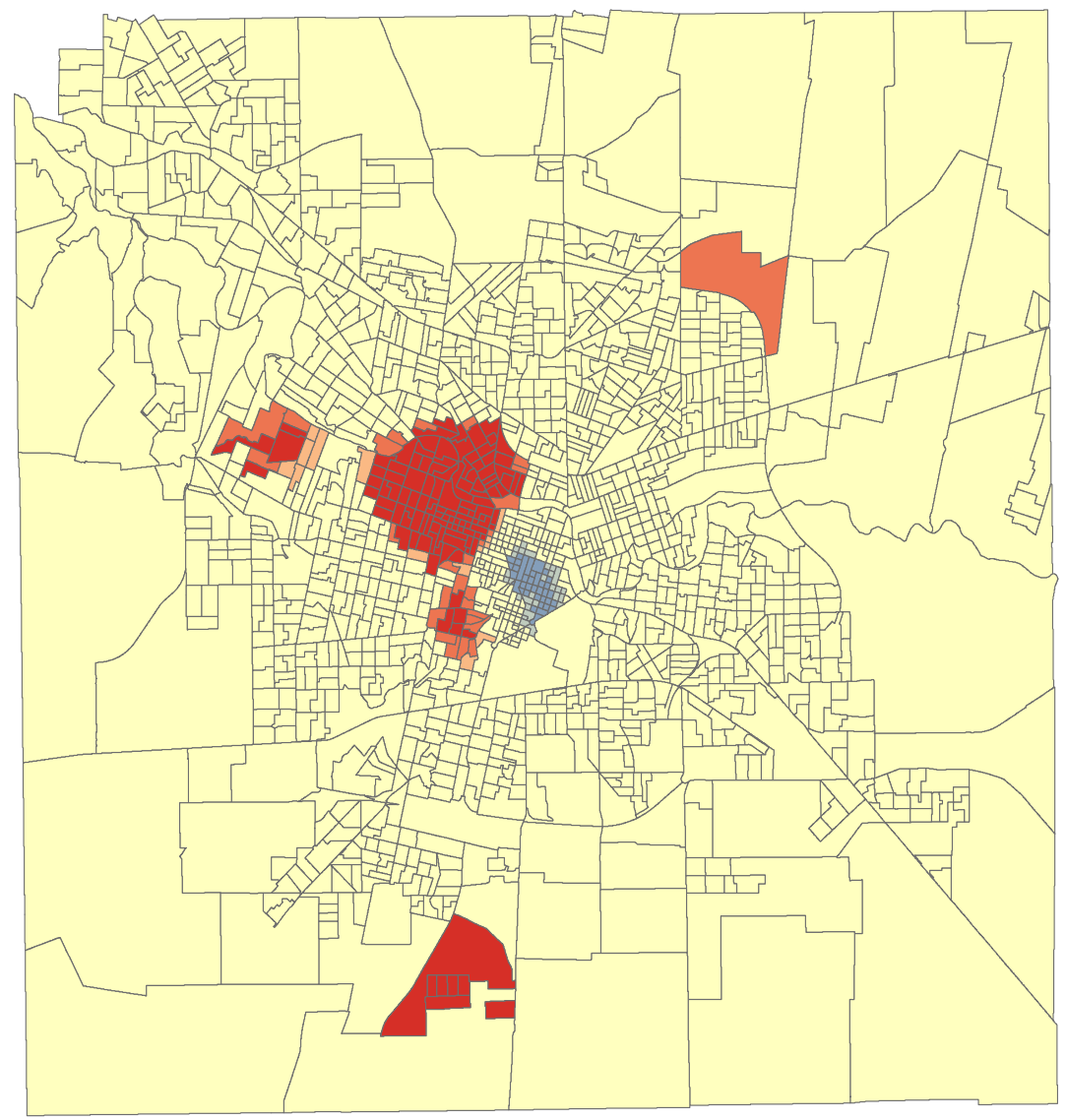

\section{Gi_Bin}

Cold Spot - 99\% Confidence

Cold Spot - 95\% Confidence

Cold Spot - 90\% Confidence

Not Significant

Hot Spot - 90\% Confidence

Hot Spot - 95\% Confidence

Hot Spot - 99\% Confidence

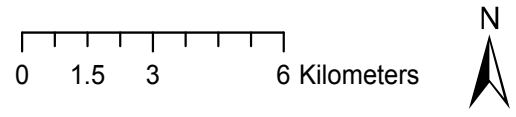

$N$

Fuente: elaboración propia con base en datos de INDEC (2010). 


\subsection{Concentración y dispersión de la población nacida en Perú}

Después de detectar los clústers de población peruana en la ciudad y de población con nivel educativo alto analizamos las principales características sociodemográficas de los peruanos agrupados y dispersos para los dos periodos censales y por género. De acuerdo a lo detallado en la sección metodológica no es posible realizar una comparación con las mismas variables. Los principales indicadores seleccionados se presentan en las tablas 2, 3, y 4.

Como puede observarse en la tabla 2 cuando consideramos los datos censales del 2001 la mayoría de los hombres que se encuentran dispersos tienen mayor tiempo de permanencia en la ciudad (69\% hace 5 años vivían en la ciudad de Córdoba y tan solo el 27\% vivían en Perú hace 5 años). En relación a las mujeres los porcentajes disminuyen, 51\% vivían en Córdoba hace 5 años y $45 \%$ vivían en Perú hace 5 años. Por el contrario, cuando consideramos los individuos peruanos que se encuentran agrupados en clústers observamos un patrón diferente: la mayoría de las personas tienen un tiempo menor de permanencia. En efecto, la proporción de hombres que vivía en Córdoba hace 5 años es de $47 \%$ y que vivía en Perú hace 5 años de $48 \%$, para el caso de las mujeres la situación muestra un patrón similar $41 \%$ y $57 \%$ respectivamente. Esto está indicando que el desplazamiento hacia la Ciudad de Córdoba de los individuos que se encuentran agrupados es más reciente y esto es más acentuado aún cuando consideramos a las mujeres.

En las actividades laborales vemos las principales diferencias en términos de género y ubicación en el espacio de la ciudad. Los hombres dispersos en la ciudad tienen mayor variabilidad en las ocupaciones que realizan sugiriendo una mayor incorporación y fusión con la población nativa. El 28\% se dedica a la construcción y el resto está dividido en comercio (15\%), industria (12\%) y servicios sociales y de salud $10 \%$. Los hombres agrupados presentan un patrón muy diferente, están más concentrados en una actividad: la construcción, es decir, la variabilidad ocupacional es menor. El caso de las mujeres es diferente, ya que tanto las que se encuentran concentradas como las dispersas trabajan en una ocupación principal: el servicio doméstico. En resumen, los hombres dispersos tienen mayor variabilidad en los patrones ocupacionales que los hombres agrupados. Para las mujeres la situación es diferente, ya que tanto las agrupadas como las dispersas tienen poca variabilidad en la ocupación que desarrollan.

Complementario a este análisis detallamos las calificaciones de las actividades que realizan los migrantes. De manera convergente a lo que ocurre con las ramas de actividad, hay diferencias entre los hombres agrupados y los dispersos pero no se observan diferencias significativas entre las mujeres agrupadas y las que se encuentran dispersas. Los hombres dispersos se concentran 
en actividades operativas en primer lugar (53\%), en segundo lugar técnicas $(15 \%)$ y no calificadas $(15 \%)$ y profesionales en tercer lugar $12 \%$. Por el contrario, los hombres agrupados se concentran en mayor proporción en actividades operativas (70\%) y no calificadas (13\%). Es decir, cuando consideramos la calificación de las actividades hay mayor variabilidad en los hombres que se encuentran dispersos que en los que se encuentran concentrados. En el caso de las mujeres la situación se mantiene estable para ambos grupos, tanto las dispersas como las agrupadas se concentran en actividades no calificadas (83\% para las primeras y $88 \%$ para las segundas respectivamente). Sin embargo, hay una mayor proporción de mujeres dispersas que están en actividades operativas y técnicas que las otras.

Finalmente detallamos dos indicadores socioeconómicos de relevancia: tenencia de obra social y aportes jubilatorios. La temporalidad diferencial en el movimiento migratorio tiene correlato con estas condiciones de incorporación. En el caso de la tenencia de obra social como puede verse en la tabla 2 observamos diferencias entre los agrupados y los dispersos. El porcentaje de hombres dispersos que tienen obra social es del 35\% y de mujeres en la misma condición del 19\%. Los individuos agrupados muestran indicadores más precarios, solo el $16 \%$ de los hombres tienen cobertura médica y solo el $9 \%$ de las mujeres las tienen. Lo destacable en ambos casos es que las mujeres en ambas situaciones se encuentran en una situación más desfavorable en términos de acceso a servicios de salud que los hombres.

La situación con los aportes jubilatorios es convergente con los análisis previos: hay mayores similitudes entre las mujeres agrupadas y dispersas que entre los hombres en ambas condiciones. Es decir, la condición de género es el rasgo en común y no la concentración o dispersión en la ciudad. Los hombres dispersos se encuentran en la mejor situación con respecto a este indicador, el 43\% tiene aportes jubilatorios. La situación para el resto de los individuos es precaria. Tan solo el 19\% de las mujeres dispersas tiene aportes jubilatorios en tanto que de los individuos agrupados solo $16 \%$ de los hombres y $9 \%$ de las mujeres tiene aportes 
Tabla 2 - Características principales por sexo de los individuos nacidos en Perú agrupados y dispersos. 2001.

\begin{tabular}{lcccc}
\hline & \multicolumn{2}{c}{ Dispersos } & \multicolumn{2}{c}{ Agrupados } \\
\cline { 2 - 5 } & Hombres & Mujeres & Hombres & Mujeres \\
\hline Tiene obra social & 35 & 19 & 16 & 9 \\
\hline Vivía en Córdoba hace 5 años & 69 & 51 & 47 & 41 \\
\hline Vivía en Perú hace 5 años & 27 & 45 & 48 & 57 \\
\hline Principales actividades & 28 & 0,1 & 50 & 0,4 \\
Construcción & 2 & 79 & 3 & 83 \\
Servicio Doméstico & 15 & 5 & 10 & 3 \\
Comercio & 12 & 2 & 10 & 2 \\
Industria & 10 & 6 & 4 & 3 \\
Serv. sociales y salud & & & & \\
\hline Calificación de las actividades & 12 & 1 & 6 & 3 \\
Profesional & 15 & 6 & 8 & 3 \\
Técnica & 53 & 8 & 70 & 98 \\
Operativa & 15 & 83 & 13 & 75 \\
No calificada & 57 & 81 & & 90 \\
\hline Sin aportes jubilatorios & & & & 3 \\
\hline
\end{tabular}

Nota: algunas variables no suman 100 porque se trata de una selección de las principales categorías. Fuente: elaboración propia con base en datos de INDEC (2010).

En la tabla 3 se presentan indicadores con base en datos censales del año 2010. Como puede observarse, la proporción de jefaturas masculinas es mayor en los hogares con al menos una persona nacida en Perú que se encuentran dispersos (72\% dispersos, 65\% agrupados). En el caso de la edad, sosteniendo la hipótesis de progresiva dispersión en el espacio urbano, podemos observar que los hogares peruanos que se encuentran dispersos tienen promedios de edad mayores que los agrupados. El nivel educativo para los hombres dispersos también es más elevado (el porcentaje con nivel educativo alto es del $45 \%$ para los dispersos en tanto que para los agrupados es de tan solo el 26\%). En el caso de las jefaturas de hogar femeninas hay un patrón común entre las que se encuentran agrupadas y dispersas en cuanto al nivel educativo: es menor en ambas situaciones en relación a los hombres. Las mujeres que se encuentran agrupadas, por su parte, concentran mayor proporción en los niveles medio y bajo. En relación a la condición de actividad es de destacar una situación común a todos los hogares, con alta actividad y donde la proporción de inactivos es mayor entre las mujeres. 
Tabla 3 - Características principales por sexo de los individuos nacidos en Perú agrupados y dispersos. 2010.

\begin{tabular}{cccccc}
\hline & \multicolumn{2}{c}{ Dispersos } & \multicolumn{2}{c}{ Agrupados } \\
\hline \multicolumn{2}{c}{} & Hombres & Mujeres & Hombres & Mujeres \\
\hline Jefe de hogar peruano & 72 & 28 & 65 & 35 \\
Edad & & 42 & 41 & 38 & 40 \\
Niv. Educativo & Bajo & 7 & 12 & 10 & 15 \\
& Medio & 49 & 51 & 64 & 60 \\
\multirow{3}{*}{ Actividad } & Alto & 45 & 37 & 26 & 24 \\
& Ocupado & 92 & 82 & 95 & 88 \\
& Desocupado & 2 & 4 & 2 & 3 \\
& Inactivo & 6 & 14 & 3 & 9 \\
\hline
\end{tabular}

Nota: algunas variables no suman 100 porque se trata de una selección de las principales categorías. Fuente: elaboración propia con base en datos de INDEC (2010).

Finalmente analizamos dos indicadores centrales en la ocupación del espacio urbano y las condiciones de alojamiento: los principales regímenes de tenencia y los tipos de viviendas. Los tabulados se presentan en la tabla 4. Aquí encontramos diferencias relevantes, para el censo del 2001 el porcentaje de hogares propietarios del terreno y la vivienda es más elevado en los que se encuentran dispersos, $49 \%$ en tanto que los otros hogares tan solo el $14 \%$ es propietario. El régimen de inquilinato es menor en los dispersos $37 \%$ en tanto que los agrupados ascienden a $78 \%$. Las principales viviendas también muestran un patrón diferencial. Los hogares dispersos mayoritariamente viven en casas, $64 \%$ en tanto que los agrupados solo el $40 \%$. La diferencia principal está en la mayor proporción de hogares agrupados que viven en una situación habitacional más precaria: en departamentos el $26 \%$ y en inquilinato $22 \%$. Para el censo del año 2010 el patrón es similar: mayor precariedad en los hogares agrupados, aunque con algunas variaciones. Por ejemplo, aumenta para los hogares peruanos agrupados en el 2010 la proporción de propietarios de vivienda y terreno (lo cual podría considerarse una mejora en las condiciones habitacionales), en el caso de los dispersos no hay diferencias significativas. En cuanto a las principales viviendas se observa para los hogares dispersos un aumento en la proporción que vive en casas (y una disminución de las piezas en inquilinato). En cuanto a los agrupados se observa un proceso similar, aumento de los hogares que viven en casas (pasa del 40\% en el 2001 al 50\% en el 2010) y una disminución de las piezas en inquilinato (pasa del $22 \%$ en el 2001 al $12 \%$ en el 2010). 
Tabla 4 - Características principales de hogares con al menos una persona nacida en Perú. Agrupados y dispersos. 2001-2010.

\begin{tabular}{cccccc}
\hline & & \multicolumn{2}{c}{ Dispersos } & \multicolumn{2}{c}{ Agrupados } \\
\hline \multirow{2}{*}{$\begin{array}{c}\text { Régimen de } \\
\text { tenencia }\end{array}$} & 2001 & 2010 & 2001 & 2010 \\
\hline Principales & Propietario vivienda y terreno & 49 & 46 & 14 & 25 \\
viviendas & Casa & 37 & 39 & 78 & 70 \\
& Departamento & 19 & 20 & 26 & 25 \\
& Pieza en inquilinato & 7 & 3 & 22 & 12 \\
& Pieza en hotel o pensión & 2 & 1 & 6 & 9 \\
\hline
\end{tabular}

Nota: algunas variables no suman 100 porque se trata de una selección de las principales categorías.

Fuente: elaboración propia con base en datos de INDEC $(2001,2010)$.

\section{Discusión y conclusiones}

El análisis empírico sugiere que existen cambios y continuidades en los patrones de asentamiento espacial de la población peruana en la Ciudad: estabilidad de la zona céntrica como polo receptor de nuevos migrantes y nuevos espacios periféricos de asentamiento. Para el último periodo del cual se tienen datos censales (2010) se observan nuevos asentamientos en zonas que no tienen concentración de población nativa con nivel educativo alto, lo que señala mutaciones en relación al periodo precedente. Hasta el año 2001 los peruanos se concentraron principalmente en zonas de población nativa con nivel educativo alto (zona centro y noroeste de la Ciudad). Sin embargo, debe matizarse una percepción binaria de la segregación (segregación/dispersión, exclusión/integración, etc.). Es decir, no necesariamente la concentración implica exclusión, como vimos, una parte de la población peruana se localiza en la zona céntrica de la ciudad y esa zona se caracteriza por acceso a servicios. Como ha sido señalado en la literatura previa muchas veces la proximidad de connacionales es una estrategia óptima de supervivencia y fortalece las redes sociales de proximidad. En el caso de la migración peruana, las investigaciones previas han señalado la importancia de las mismas. El análisis espacial sugiere, sin embargo, que otros segmentos de población peruana para el año 2010 se encuentran en una zona periférica y por lo tanto bajo los mismos condicionantes que la población nativa en esos mismos espacios urbanos (presión del mercado inmobiliario, falta de infraestructura y transporte, servicios, etc.).

Después de detallar los patrones espaciales de la población peruana para ambos momentos censales se analizó comparativamente los peruanos agrupados en clústers y aquellos dispersos. La evidencia empírica es consistente con un proceso de progresiva dispersión en el espacio urbano e incorporación 
espacial para los migrantes más antiguos. Sin embargo, debemos señalar una serie de elementos que complejizan ese proceso:

a) la existencia de dos macro-cohortes de migrantes peruanos en la Ciudad, con composiciones de clase diferenciales. Es decir, aquellos migrantes peruanos que se encuentran dispersos tenían otra composición de clase y es difícil establecer comparaciones con los migrantes más recientes cuyo asentamiento en la ciudad además de ser más reciente tiene otras características.

b) La estabilidad del clivaje de género como elemento que estructura las modalidades de incorporación espacial en la ciudad. Como observamos en el apartado previo, independientemente de la condición de concentración/dispersión, las mujeres se encuentran en una situación socioeconómica más precaria.

c) La dispersión en el espacio urbano no implica una convergencia con los patrones ocupacionales de la población de destino.

d) La interacción entre los procesos de segregación propios de la ciudad y la población nativa con los procesos de incorporación de la población de origen peruano. Como observamos, para el último periodo censal se observan nuevos asentamientos en una zona periférica de la ciudad.

Nuestro análisis es en términos estructurales, es decir, sobre las condiciones objetivas de segregación y sobre los patrones espaciales y sociodemográficos de los migrantes peruanos, por lo tanto provee una visión estática del fenómeno. Sin embargo, permite evaluar e identificar las condiciones estructurales en la cuales los migrantes y sus comunidades (en términos de interfase) transitan y comparten la ciudad. Es necesario complementar este abordaje con trabajos que den cuenta de la multiplicidad de circulaciones y apropiaciones del espacio y la ciudad. De esta manera complejizar la lógica de centro/periferia de la ciudad y articular la dimensión objetiva con el estudio de la experiencia social y urbana de los migrantes en Córdoba.

\section{Bibliografía}

ALBA, Richard; NEE, Victor. Remaking the American mainstream: assimilation and contemporary immigration. Cambridge, Mass.: Harvard Univ. Press, 2003.

BAIADERA ALVITES, Angélica. Entre redes sociales. Prácticas y representaciones de la migración peruana. Córdoba: EDUVIM, 2015.

BOLOGNA, Eduardo; FALCÓN, María del Carmen. Migración sur-sur: Factores relacionales e inserción segmentada de la población boliviana y peruana en la ciudad de Córdoba (Argentina). Estudios Demográficos y Urbanos, v. 31, n. 3, 2016, p. 729-773. 
BOLOGNA, Eduardo; GÓMEZ, Pablo Sebastián; MORALES, Susana; PLAZA, Valeria. El derecho a la ciudad en cuestión: segregación residencial y experiencias de circulación de jóvenes en la Ciudad de Córdoba, Argentina. Quid 16. Revista del Área de Estudios Urbanos, v. 6, 2017.

BOLT, Gideon; KEMPEN, Ronald van. Escaping poverty neighbourhoods in the Netherlands. Housing, Theory and Society, v. 20, n. 4, 2003, p. 209-222.

BOLT, Gideon; KEMPEN, Ronald van. Ethnic Segregation and Residential Mobility: Relocations of Minority Ethnic Groups in the Netherlands. Journal of Ethnic and Migration Studies, v. 36, n. 2, 2010, p. 333-354.

BOLT, Gideon; ÖZÜEKREN, A. Sule; PHILLIPS, Deborah. Linking Integration and Residential Segregation. Journal of Ethnic and Migration Studies, v. 36, n. 2, 2010, p. 169-186.

CERRUTTI, Marcela; MAGUID, Alicia. Inserción laboral e ingresos de los migrantes de países limítrofes y peruanos en el gran Buenos Aires. Notas de población, v. 83, 2007, p. 75-98.

DEVOTO, Fernando; OTERO, Hernán. Veinte años después: una lectura sobre el Crisol de razas, el pluralismo cultural y la historia nacional en la historiografía argentina. Estudios Migratorios Latinoamericanos, v. 17, n. 50, 2003, p. 181-228.

FALCÓN, María del Carmen; BOLOGNA, Eduardo. Migrantes antiguos y recientes: Una perspectiva comparada de la migración peruana a Córdoba, Argentina. Migraciones Internacionales, v. 7, n. 1, 2013, p. 235-266.

FREEMAN, Lance. Does Spatial Assimilation Work for Black Immigrants in the US? Urban Studies, v. 39, n. 11. 2002.

FREEMAN, Lance. Minority Housing Segregation: A Test of Three Perspectives. Journal of Urban Affairs, v. 22, n. 1, 2000, p. 5-35.

GALSTER, George C. The Evolving Challenges of Fair Housing Since 1968: Open Housing, Integration, and the Reduction of Ghettoization. Cityscape. Journal of Policy Development and Research, v. 4, n. 3, 1999, p. 123-138.

GETIS, Arthur; ORD, J. Keith. The Analysis of Spatial Association by Use of Distance Statistics. Geographical Analysis, v. 24, n. 3, 1992, 189-206.

GÓMEZ, Pablo Sebastián; SANCHEZ SORIA, David. Concentración, dispersión y características sociodemográficas en la incorporación espacial de la migración peruana en la Ciudad de Córdoba, Argentina. Población y Salud en Mesoamérica [online], v. 14, n. 1, 2016.

GORDON, Milton M. Assimilation in American Life: The Role of Race, Religion and National Origins. New York: Oxford University Press, USA, 1964.

INDEC. Censo Nacional de Población, Hogares y Viviendas del año 2001. Instituto Nacional de Estadística y Censos, 2001.

INDEC. Censo Nacional de Población, Hogares y Viviendas del año 2010. Instituto Nacional de Estadística y Censos, 2010.

JUNG, Moon-Kie. The Racial Unconscious of Assimilation Theory. Du Bois Review: Social Science Research on Race, v. 6, n. 2, 2009, 375-395. 
KEMPEN, Ronald van; ÖZÜEKREN, Sule. Ethnic Segregation in Cities: New Forms and Explanations in a Dynamic World. Urban Studies, v. 35, n. 10, 1998, p. 1631-1656.

KIVISTO, Peter. What is the canonical theory of assimilation?. Journal of the History of the Behavioral Sciences, v. 40, n. 2, 2004, 149-163.

MAGLIANO, María José; PERISSINOTTI, María Victoria; ZENKLUSEN, Denise. Mujeres bolivianas y peruanas en la migración hacia Argentina: especificidades de las trayectorias laborales en el servicio doméstico remunerado en Córdoba. Anuario Americanista Europea, v. 11, 2013, p. 71-91.

MARCOS, Mariana; MERA, Gabriela. Migrantes internacionales en la Aglomeración Gran Buenos Aires: un análisis cuantitativo de su distribución espacial. Cuadernos Geográficos, v. 54, n. 1, 2015, p. 257-282.

MARENGO, Cecilia; MONAYAR, Virginia. Crecimiento urbano e informalidad residencial: El caso Nuestro Hogar III en la periferia de Córdoba, Argentina. Cuaderno urbano, v. 13, n. 13, 2012, p. 7-25.

MASSEY, Douglas; DENTON, Nancy. The Dimensions of Residential Segregation. Social Forces, v. 67, n. 2, 1988, p. 281-315.

MERA, Gabriela; MARCOS, Mariana. Cartografías migratorias urbanas. Distribución espacial de la población extranjera en la Ciudad de Buenos Aires (2010). Geograficando [online], v. 11, n. 1. 2015. Disponible en: < http://www. geograficando.fahce.unlp.edu.ar/article/view/Geov11n01a04>.

MERA, Gabriela; MARCOS, Mariana; VIRGILIO, María Mercedes Di. Migración internacional en la Ciudad de Buenos Aires: un análisis socioespacial de su distribución según tipos de hábitat. Estudios Demográficos y Urbanos, v. 30, n. 2, 2015, p. 327-367.

MOLINATTI, Florencia. Segregación residencial socioeconómica en la ciudad de Córdoba (Argentina): Tendencias y patrones espaciales. Revista INVI, v. 28, n. 79, 2013, p. 61-94.

MORENO, Martín. La distribución espacial de las comunidades peruanas en los Estados Unidos. Debates en Sociología, n. 36, 2011. Disponible en: < http:// revistas.pucp.edu.pe/index.php/debatesensociologia/article/view/2170>.

MUSTERD, Sako. Segregation and integration: A contested relationship. Journal of Ethnic and Migration Studies, v. 29, n. 4, 2003, p. 623-641.

NEWBOLD, K. Bruce. Immigration, Spatial Assimilation, and Segmented Paths within the Metropolis. Geography Research Forum, v. 23, 2003, p. 56-77.

ORD, J. Keith; GETIS, Arthur. Local Spatial Autocorrelation Statistics: Distributional Issues and an Application. Geographical Analysis, v. 27, n. 4, 1995, p. 286-306.

OTERO, Hernán; PELLEGRINO, Adela. Compartir la ciudad. Patrones de residencia e integración de inmigrantes en Buenos Aires y Montevideo durante la inmigración masiva. In OTERO, Hernán (dir.). El mosaico argentino. Modelos y representaciones del espacio y de la población, siglos XIX-XX. Buenos Aires, Argentina: Siglo XIX Editores, 2004. 
PARK, Robert. The Urban Community as a Spatial Pattern and a Moral Order. In BURGESS, Ernest; PARK, Robert. The Urban Community. Chicago: University of Chicago Press, 1926, p. 3-18.

SOUTH, Scott J.; CROWDER, Kyle; CHAVEZ, Erick. Migration and spatial assimilation among U.S. latinos: Classical versus segmented trajectories. Demography, v. 42, n. 3, 2005, p. 497-521.

SZUCHMAN, Mark D. Mobility and Integration in Urban Argentina: Córdoba in the Liberal Era. Austin: University of Texas Press, 1980.

Recibido para publicación en 11.11.2016

Aceptado para publicación en 11.05.2017

Received for publication in November $11^{\text {th }}, 2016$

Accepted for publication in May $11^{\text {th }}, 2017$

ISSN impresso 1980-8585

ISSN eletrônico 2237-9843

http://dx.doi.org/10.1590/1980-85852503880005009 